\title{
INTEGRATED RESEARCH METHODS \\ (MIXED METHOD RESEARCH DESIGN)
}

\section{ABSTRACT}

\author{
Dr. R. Danasu
}

Research designs are procedures for collecting, analyzing, interpreting, and reporting data in research studies. They represent different models for doing research, and these models have distinct names and procedures associated with them. Research designs are useful, because they help guide the methods decisions that researchers must make during their studies and set the logic by which they make interpretations at the end of their studies. Once the researcher has identified that the research problem calls for a mixed methods approach and reflected on the philosophical and theoretical foundations of the study, the next step is to choose a specific design that best fits the problem and the research questions in thestudy.

Key Words: Mixed Research Method, Research Design.

\section{Introduction}

The trend towards evidence-based healthcare requires healthcare professionals to base their current clinical practice on scientifically derived findings rather than on tradition, intuition, assumption and precedent as has happened in the past. In addition, there is an increasing emphasis placed upon the value of research, specifically the need for members of all clinical professions to be able to undertake and appreciate research and apply research topractice. $^{1}$

In Nursing, research has not always been related to practice and there are large areas of work for which there is no reliable evidence. One reason for this may be the qualitative / quantitative divide in nursing research. As more research is carried out to widen the evidence base for care delivery, there is a need for a more flexible approach. This flexibility is likely to include using a variety of different research methods, sometimes in combination with each other.
Recently, Nursing Research was dominated by Quantitative studies. Consistent with the overall expansion of Nursing Research inquiry and with increased appreciation of methodologic pluralism, Qualitative studies have gained considerable ground since the 1980s. A growing trend is the blending of Qualitative and Quantitative data within single studies or coordinated clusters of studies. $^{2}$

\section{Definition of Integrated Research Methods (Mixed Method Research Design)}

A mixed methods research design is a procedure for collecting, analyzing,and -mixing\| both quantitative and qualitative research and methods in a single study to understand a research problem.

\section{Rationale for Mixed Method Research Design}

The dichotomy between Quantitative and Qualitative data represents the key 
epistemologic and methodologic distinction within the Social, behavioral, and health sciences. There are many advantages of combining various types of data in an investigation.

1. Complementarity

2. Enhanced Theoretical Insights

3. Incrementality

4. Enhanced validity

5. Creating newFrontiers

\section{Complementarity}

Researchers address problems with methods and measures that are fallible. By integrating different methods and modes of analysis, the weakness of a single approach may be diminished or overcome. Quantitative studies are often strong in generalizability, precision and control over extraneous variables. Sometimes the validity of such research is called into question. By introducing tight controls, quantitative studies may fail to capture situational context. Qualitative Research, by contrast has strength and weakness that are diametrically opposite. The strength of qualitative research lies in its flexibility and its potential to yield insights into the true nature of complex phenomena through indepth scrutiny. However the strength and weaknesses of quantitative and qualitative data are complementary. Combined shrewdly in a single study, qualitative and quantitative data can supply each other's lack.

\section{Enhanced Theoretical Insights}

Qualitative and Quantitative research constitute alternative ways to viewing and interpreting the word. These alternatives are not necessarily correct or incorrect: rather, they reflect and reveal different aspects of reality. To be maximally useful, nursing research should strive to understand these multiple aspects. We believe that the blending of qualitative and quantitative data in a single analysis can lead to insights on these multiple aspects that might be unattainable without such integration.

\section{Incrementality}

Qualitative methods are well suited to exploratory or hypothesis-generating research early in the development of a problem area, and quantitative methods are needed as the problem area matures for the purposes of verification. The need for exploration and in-depth insights is rarely confined to the beginning of an area of research inquiry, and subjective insights may need to be evaluated early and continually. Thus progress in developing a body of evidence for nursing practice tends to be incremental and rely on multiple feedbackloops.

\section{Enhanced validity}

Another advantage of designing multimethod research lies in the potential for enhancing the validity of study findings. When researchers hypotheses or models are supported by multiple and complementary types of data, they can be more confident about the validity of the results. The use of single approach can leave the study vulnerable to at least one and often more than one validity problem. The integration of Qualitative and Quantitative data can provide better opportunities fortesting 
alternative interpretations of the data, for examining the extent to which the context helped to shape the results, and for arriving at convergence in tapping aconstruct.

\section{Creating New Frontiers}

Researchers sometimes find that Qualitative and Quantitative data are inconsistent with each other. This lack of congruity - when it happens in the context of a single investigation can lead to insights that can push a line of inquiry further than would otherwise have been possible. ${ }^{3}$

\section{Steps for Conducting a Mixed Method Research design}

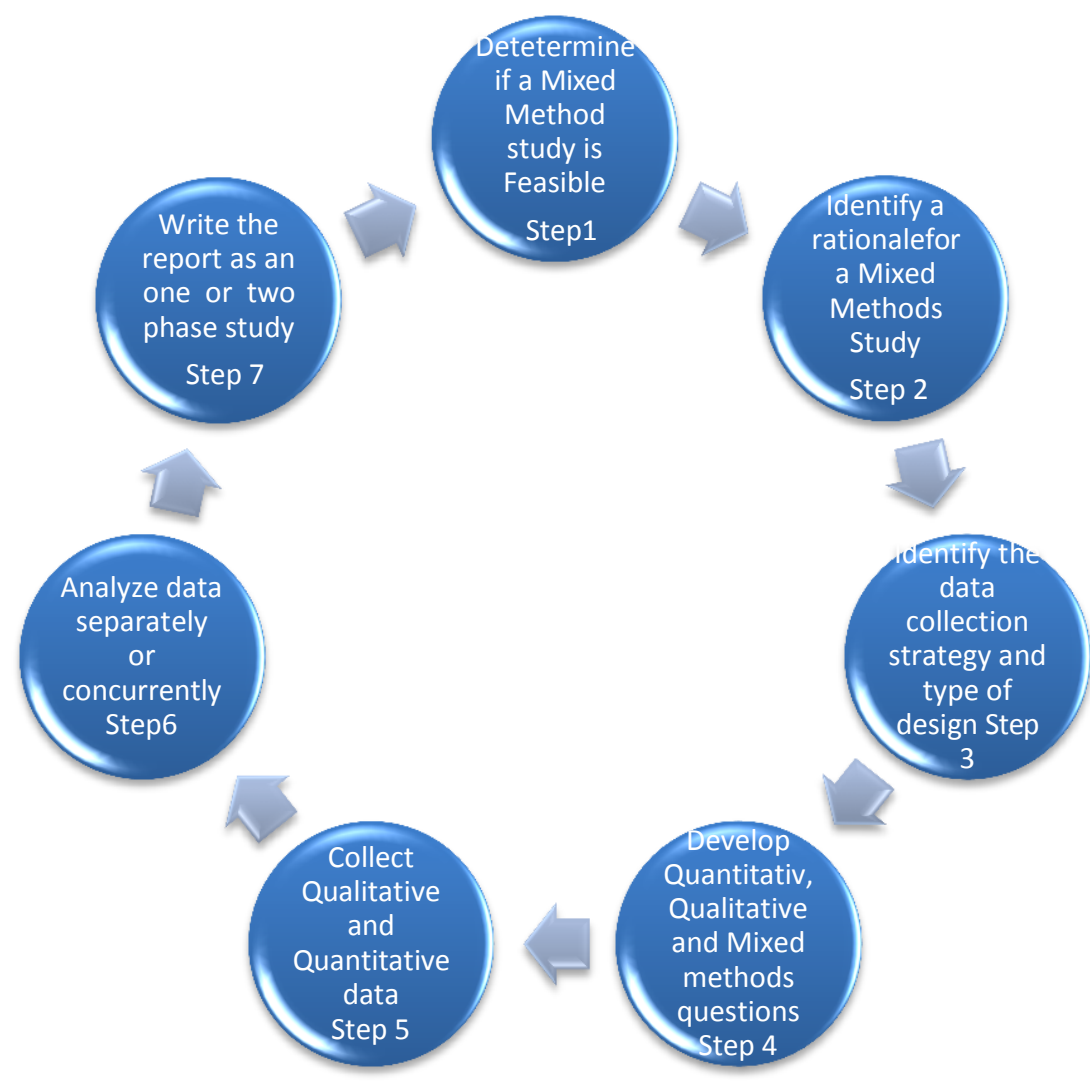

\section{Six Major Mixed Method Research design}

\section{SequentialExplanatory}

- Characterized by: Collection and analysis of quantitative data followed by a collection and analysis of qualitativedata.
- Purpose: To use qualitative results to assist in explaining and interpreting the findings of a quantitativestudy. 


\section{SequentialExploratory}

- Characterized by: An initial phase of qualitative data collection and analysis followed by a phase of quantitative data collection andanalysis.

- Purpose: To explore a phenomenon. This strategy may also be useful when developing and testing a newinstrument

\section{SequentialTransformative}

- Characterized by: Collection and analysis of either quantitative or qualitative data first. The results are integrated in the interpretationphase.

- Purpose: To employ the methods that best serve a theoreticalperspective.

\section{ConcurrentTriangulation}

- Characterized by: Two or more methods used to confirm, cross-validate, or corroborate findings within a study. Data collection isconcurrent.

- Purpose: Generally, both methods are used to overcome a weakness in using one method with the strengths ofanother.

\section{ConcurrentNested}

- Characterized by: A nested approach that gives priority to one of the methods and guides the project, while another is embedded or-nested.\|

- Purpose: The purpose of the nested method is to address a different question than the dominant or to seek information from differentlevels.

\section{ConcurrentTransformative}

- Characterized by: The use of a theoretical perspective reflected in the purpose or research questions of the study to guide all methodologicalchoices.

- Purpose: To evaluate a theoretical perspective at different levels ofanalysis. ${ }^{4}$

\section{What are some Strengths?}

- Can be easy to describe and toreport.

- Can be useful when unexpected results arise from a priorstudy.

- Can help generalize, to a degree, qualitative data.

- Helpful in designing and validating an instrument.

- Can position research in a transformative framework.

\section{What are some weaknesses?}

- Timerequired.

- Resolving discrepancies between different types ofdata.

- Some designs generate unequalevidence.

- Can be difficult to decide when to proceed in sequentialdesigns.

- Little guidance on transformativemethods. ${ }^{5}$

\section{Conclusion}

In conclusion, mixed research is the newest research paradigm in educational research. It offers much promise, and we expect to see much more methodological work and discussion about mixed research in the future as more researchers and book authors become aware of this important approach to empirical research. 


\section{Reference:}

1 Denise F. Polit, -Nursing Research Principles and Methods, $7^{\text {th }}$ Edition, Lippincott.

2 Patricia Benner, Nursing Research Designs and Methods, $1^{\text {st }}$ edition, Churchill Livingstone
3 Bharat Pareek, A Text book of Nursing Research \& Statistics, $3^{\text {rd }}$ Edition, PeeVee.

4 Sunanda S. Ray Chowdhury, Text Book of Nursing Research, $1^{\text {st }}$ edition, Kumar Publications.

5 Nancy Burns and Susan K. Grove, Understanding Nursing Research, $4^{\text {th }}$ edition,Elsevier.

\section{Sad Demise to Dr. APJ Abdul Kalam}

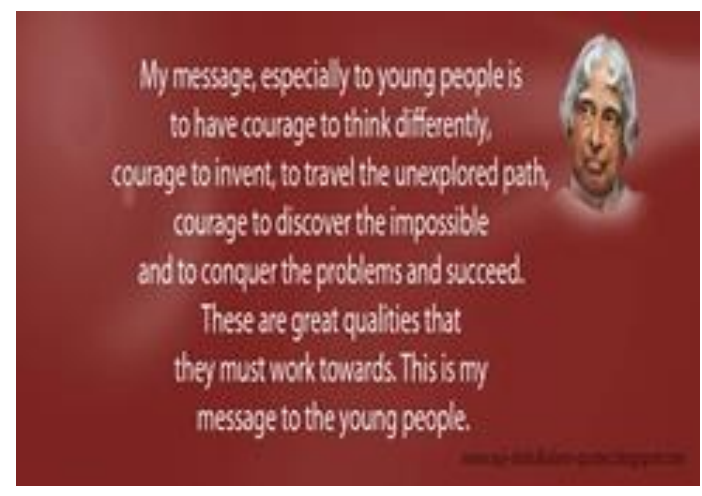

$1931-2015$

We lost a great hero and friend of children and youth of our times. Dr. APJ Abdul Kalam was a visionary full of energy and enthusiasm. We feel deeply grieved by the sad news about the unexpected demise of former President. Dr. APJ AbdulKalam.

Dr. Kalam was the most secular soul we had in our public realm...a leader who implored us to dream beyond the clouds, a visionary who put science and common sense before blind faith and religion, a true blue crusader for the awakening of a lethargic nation.....A man with profound knowledge, inspiration to entire nation, his teachings and ideas will long live in our hearts. His death is an irreparable loss to our nation which created a big void impossible to fill. We revere in the bright memory of him. Paying our tribute to the departed soul and may his soul rest in peace.

Dr. Kalam, when you became the President, you gave the word 'hope' a new meaning for Indians... Today, we have lost a great leader who inspired our young minds to feel that we live in the greatest nation on earth and that each one of them can achieve great things... May the creator lead you to heaven. Let us all salute him. Let his soul rest in peace. 